\title{
A simple model for submaximal exchange through the Strait of Gibraltar*
}

\author{
JAVIER DELGADO, JESÚS GARCÍA LAFUENTE and JUAN MIGUEL VARGAS \\ Departamento de Física Aplicada II, Universidad de Málaga, Campus Teatinos, 29071 Málaga, Spain. \\ E-mail: glafuente@ctima.uma.es
}

\begin{abstract}
SUMMARY: This work presents a simple two-layer model for the water exchange through the Strait of Gibraltar. The model is inspired by the work of Bormans and Garrett (1989) and focus on the case of submaximal exchange with an unique hydraulic control at the section of minimum cross-area. The first part describes the model and justifies the assumed simplifications. Some aspects regarding its performance are also analysed. The second part applies the model to interpret the seasonal signals in the exchanged flows that were observed during the Canary Islands Azores Gibraltar Observations (CANI$\mathrm{GO})$ project. These signals are compatible with submaximal exchange, which suggests that the oceanographic conditions in the nearby Alboran Sea are able to influence the exchange via interface depth variations.
\end{abstract}

Key words: Gibraltar Strait, hydraulic control, barotropic forcing, interface oscillations.

\section{INTRODUCTION}

The Strait of Gibraltar is the only natural connection between the Mediterranean Sea and the open oceans. It has a negative water balance: Evaporation, $\mathrm{E}$, is greater than precipitation over the basin plus river discharge, $\mathrm{P}$, producing a net fresh water flux (E-P) toward the atmosphere.

The mean exchange through the Strait of Gibraltar is density driven in response to this large evaporation, with Atlantic water flowing into the Mediterranean Sea at surface and saltier and denser Mediterranean water flowing out at depth. The exchange is influenced by other forces like atmospheric forcing, tides, earth rotation, flow-topography interactions (Candela et al., 1989; Armi and Farmer, 1988; Bryden and Kinder, 1991 (hereafter BK91); Garcia Lafuente et al., 2000), which produce fluctuations with periods

\footnotetext{
*Received August 31, 2000. Accepted March 23, 2001.
}

ranging from the few minutes associated with the short internal waves of near Brünt Väisälä frequency (Watson and Robinson, 1990), up to inter-annual variability, which is not well known yet.

Many of the recent papers on the exchange through the Strait use the two-layer hydraulic control theory as an appropriate conceptual model to interpret the observations. This theory was developed by Farmer and Armi (1986, hereafter FA86) for a strait with a sill and a contraction. They showed that maximal exchange needs the existence of two control sections, the one of minimum width (contraction) and that of minimum depth (sill), in which the composite Froude number is critical:

$$
G^{2}=F_{1}^{2}+F_{2}^{2}=\frac{u_{1}^{2}}{g^{\prime} h_{1}}+\frac{u_{2}^{2}}{g^{\prime} h_{2}}=1
$$

Submaximal exchange occurs when one (or both) of these sections disappear (FA86). The limit situation between these two types of exchange, the 


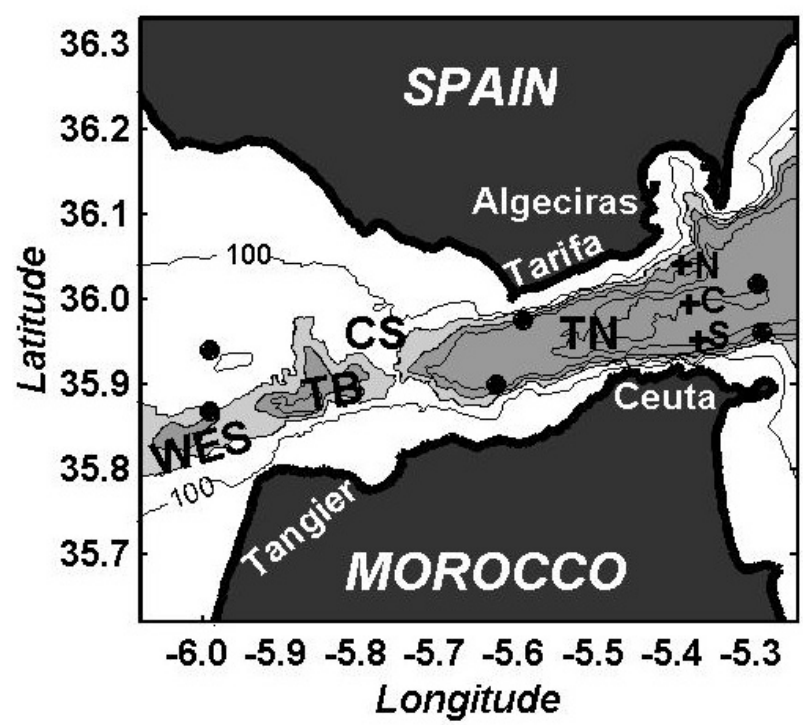

FIG. 1. - Map of the Strait of Gibraltar showing the location of the most outstanding topographic features. From left to right they are: West Espartel Sill (WES), Tangier Basin (TB), Camarinal Sill (CS) and Tarifa Narrows (TN). Crosses labelled "N","C", and "S" at the eastern section indicate the samplings sites occupied during CANIGO from which the seasonal signals in the exchanged flows were estimated in GLD01.

so-called marginally submaximal exchange (Bormans and Garrett, 1989, hereafter BG89), happens when one of the control sections is about to be lost. It produces the same amount of exchanged flows as the strictly maximal situation and can be treated like this solution in many ways. However, the shape of the interface along the strait under marginally submaximal exchange is similar to that of the submaximal exchange (BG89).

The theory is of direct applications to the Strait of Gibraltar, which has very distinctive contraction and sill sections (see Fig. 1). BK91 applied this theory together with conservative laws for salt and water within the Mediterranean Sea in order to give indirect estimates of the net evaporation within the basin. BG89 extended AF86's theory to include physically important effects such as non-rectangular cross section, friction and barotropic fluctuations. This modified model is revisited here to explore the strictly submaximal exchange in order to interpret recent observations taken at the eastern entrance of the Strait of Gibraltar between October 1995 and May 1998 within the Canary Islands Azores Gibraltar Observations (CANIGO) project. These currentmeter observations were analysed by García Lafuente et al. (2001, hereafter GLD01), who found experimental evidence that the flow was not maximal during the fieldwork. This conclusion was supported by a) the low values of the composite Froude number at the observation site
$\left(G^{2} \approx 0.3\right)$ and b) the existence of uncoupled low frequency signals for the inflow, $\mathrm{Q}_{1}$, and the outflow, $Q_{2}$. These questions were addressed in GLD01 and are revised in more detail by the simple model that we present here.

\section{MODEL DESCRIPTION}

The model is a version of that in BG89 with a more detailed topography of the Strait. While BG89 restrict to the analysis of the maximal and marginally submaximal situations (both requiring two control sections at the sill and the contraction, respectively), we explore the strictly submaximal solutions.

We assume a two-layer model of densities $\rho_{1}$, and $\rho_{2}$, and flow rates $Q_{1}$ and $Q_{2}$. Sub-index 1 (2) refer to the upper (lower) layer and positive values of $Q_{\mathrm{k}}$ indicate flow towards the Mediterranean. Thus, $Q_{2}$ will be negative unless a strong enough positive barotropic fluctuation is superposed to the exchange. The unidimensional steady momentum equation for either layer, using the hydrostatic approximation in a non-rotating earth, with z-axis vertically upward ( $z=0$ at $900 \mathrm{~m}$ depth) and $\mathrm{x}$-axis, oriented along-strait, positive toward the Mediterranean Sea $(x=0$ at Camarinal sill, hereafter CS, see Fig. 1), are

$$
\begin{gathered}
u_{1} \frac{\partial u_{1}}{\partial x}+g \frac{\partial \eta_{1}}{\partial x}=-F_{i 1}-F_{b 1} \\
u_{1} \frac{\partial u_{1}}{\partial x}+\frac{\rho_{1}}{\rho_{2}} \frac{\partial\left(\eta_{1}-\eta_{2}\right)}{\partial x}+g \frac{\partial \eta_{2}}{\partial x}=-F_{i 1}-F_{b 1}
\end{gathered}
$$

where $u_{k}, k=1,2$, are the velocities of each layer, $\eta_{1}$ and $\eta_{2}$ are the co-ordinate of the sea surface and the interface, respectively (see Fig. 2 for nomenclature). Right hand side terms are frictional coupling between layers (subindex "i") and between layer and bottom and/or walls (subindex "b"). They are approximated by quadratic laws of the form

$$
\begin{gathered}
F_{i, k}=C_{i}\left|u_{1}-u_{2}\right|\left(u_{1}-u_{2}\right) h_{k}^{-1} \\
F_{b, k}=C_{b}\left|u_{k}\right| u_{k} d_{k}
\end{gathered}
$$

where $C_{i}$ and $C_{b}$ are drag coefficients for interfacial and bottom friction, respectively, $k=1,2$, indicates the layer on which friction is acting, $h_{k}$ is the effective thickness of layer-k, estimated as $A_{k} / W_{\text {int }}, A_{k}$ being the cross area of layer- $k$ and $W_{\text {int }}$ the width of the interface, and $d_{k}$ is the length of the solid bound- 


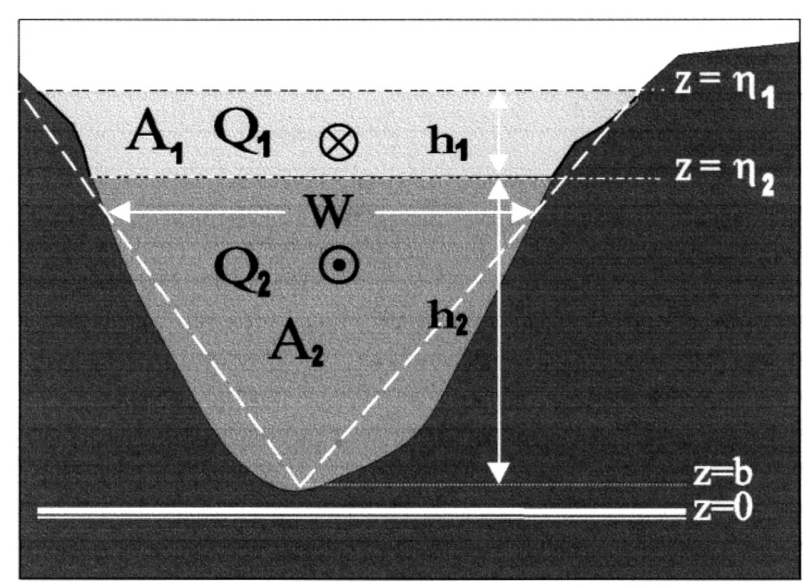

FIG. 2. - Sketch of the cross-area (dark shadow) and the equivalent triangular section (white dashed line) of the Strait of Gibraltar. Letters indicate the parameters and variables used in the model.

ary of layer- $k$ divided by the cross area $A_{k}$. These two equations are similar as Equations 6 in BG89.

Continuity equations are:

$$
\begin{aligned}
u_{1} A_{1} & =Q_{1} \\
u_{2} A_{2}=Q_{2} & =Q_{0}-Q_{1}
\end{aligned}
$$

where $A_{1}(x)$ and $A_{2}(x)$ are the cross-areas for $Q_{1}$, and $Q_{2}$, respectively, and $Q_{0}$ is the net barotropic flow.

Subtraction of the two momentum equations, and using the continuity equation and the rigid lid approximation (which is justify as far as $g^{\prime} \equiv g\left(\left(\rho_{2}-\right.\right.$ $\left.\left.\rho_{1}\right) / \rho_{2}\right)<<g$ ) yield the starting model equation:

$$
\frac{\partial}{\partial x}\left[\frac{Q_{1}^{2}}{2 A_{1}^{2}}-\frac{Q_{2}^{2}}{2 A_{2}^{2}}-g^{\prime} \eta_{2}\right]=-\left(F_{b 1}-F_{b 2}\right)-\left(F_{i 1}-F_{i 2}\right)
$$

This equation will be integrated considering that the flow is hydraulically controlled at CS, an assumption that is justified by the fact that the Mediterranean outflow sinks at depths of around $1000 \mathrm{~m}$ in the Atlantic Ocean (Garrett, 1996; Baringer and Price 1997). With a realistic triangular geometry for the cross-strait sections, Equation 1 at CS yields:

$$
\frac{Q_{1}^{2}}{g^{\prime} A_{1 S}^{3}}+\frac{Q_{2}^{2}}{g^{\prime} A_{2 S}^{3}}=\frac{\left(\eta_{1 S}-b_{S}\right)}{W_{S}\left(\eta_{2 S}-b_{S}\right)}
$$

where $W_{s}$ is the surface width at CS section, and $b_{s}$ is the bottom elevation above the reference level. We will take the Strait topography reviewed by BK91 who adjusted the section width in order to match the actual cross areas preserving the actual height, in contrast to BG89's work, who used actual widths. This change produces a reduction of $10 \%$ in the cross-areas and, hence, an elevation of the interface throughout.

Equation 4 can be integrated given $Q_{1}, Q_{2}$, and the initial position of the interface at CS, which would be obtained from 5 . The numerical integration has been carried out using a finite difference predictor-corrector scheme (Milne modified by Hamming, see Chapra and Canale (1988) for details) with up to 500 integration intervals along the axis of the Strait when the solution was close to the maximal exchange. The number of steps was reduced to 200 when the solution was clearly submaximal. This scheme has been adopted in order to avoid problems arising from the boundary condition $G^{2}=1$ in CS. The Equation 4 could be rewritten in terms of $G^{2}$ for triangular geometry as

$$
g^{\prime}\left(G^{2}-1\right) \frac{\partial \eta_{2}}{\partial x}=(\ldots) \frac{\partial W}{\partial x}+(\ldots) \frac{\partial b}{\partial x}+(\ldots) \frac{\partial\left(\frac{w}{b}\right)}{\partial x}
$$

where (...) stands for non-zero continuous positive functions. Sections in which $G^{2}=1$ (i.e., control sections) need a zero right hand side term (or very close to zero in the numerical scheme) in order to have a meaningful solution for the interface depth. Otherwise, the solution for $\eta_{2}$ is meaningless. The starting section for numerical integration is one of $G^{2}=1$ (Eq. 5) by prescription. Thus, attention must be paid at this initial stage to prevent errors from growing up and to invalidate the output.

\section{MODEL ANALYSIS}

In the most general case three parameters must be prescribed to solve Equations 4 and 5. They are, for instance, $Q_{1}, Q_{2}$ and $\Delta \rho$ (that is, $g^{\prime}$ ). Another possibility that is more interesting is to prescribe the net barotropic flow $Q_{0}$ instead of, says, $Q_{2}$. In the case of maximal or marginally submaximal exchange the flow is also critical at the contraction of Tarifa (TN), which provides a second condition that reduces the number of prescribed parameters to 2 , usually $Q_{0}$ and $\Delta \rho$. Moreover, if we assume a constant density contrast (we ignore any seasonal or low frequency signal in $\Delta \rho$ ), the flows are fully determined just prescribing the net barotropic flow. We explore the strictly submaximal exchange and do allow for signals in $\Delta \rho$. Thus, we handle three independent para- 


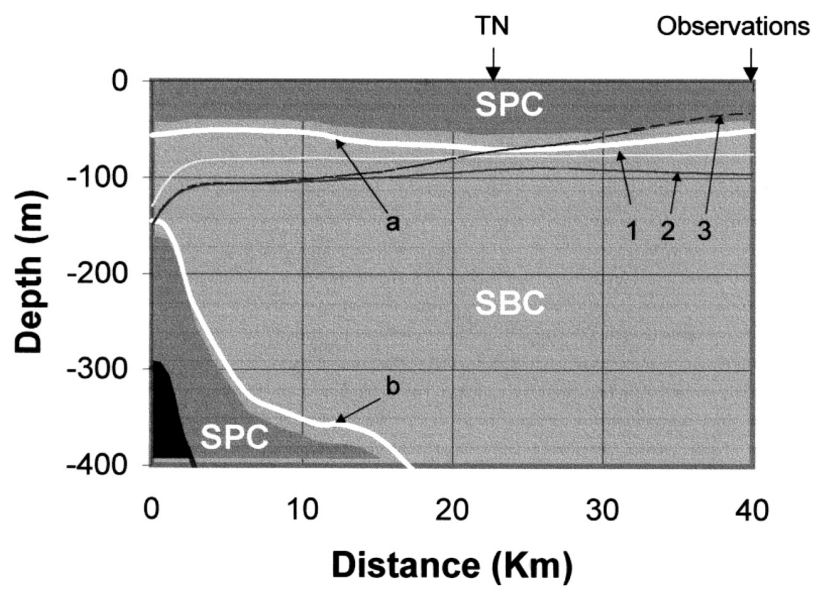

FIG. 3. - Diagram showing the mathematically permitted regions of supercritical (SPC, dark shadowed areas) and subcritical (SBC, light shadowed area) flows for the values mentioned in the text. Black area is the bottom sea. Thick line labelled a (b) is the new boundary of the upper (lower) SPC region in the case that $\mathrm{Q}_{1}\left(\mathrm{Q}_{2} \mathrm{I}\right)$ increases. White line labelled " 1 " would represent the interface depth for a submaximal exchange in which no control sections exist. Grey line labelled " 2 " is for a submaximal situation with control at the sill in the case that the lower SPC area is bound by line "b". Grey line labelled " 3 " is for a maximal situation in the case that the SPC and SBC regions are bound by lines " $b$ " (lower) and "a" (upper) respectively. TN indicates the position of Tarifa Narrows. The eastern section in which observations were made is also indicated.

meters ( 3 degrees of freedom). The analysis of this type of exchange is more complex.

Time-dependence is not included in Equations 4 and 5. We are interested in analysing low frequency variability. At this time scale, the time it takes a signal to go through the Strait is much less than the time scale of the fluctuation so the interface adjusts almost instantaneously to the new situation. Thus, the time dependence can be introduced by means of the parameters of the model, $Q_{1}(t), Q_{2}(t)$ and $\Delta \rho(t)$.

\section{A graphical tool to analyse the exchange}

The composite Froude number is the main parameter in order to analyse the stage of the exchange. For triangular cross section geometry it can be written as:

$$
G^{2}=\left(\frac{Q_{1}^{2}}{g^{\prime} A_{1}^{3}}+\frac{Q_{2}^{2}}{g^{\prime} A_{2}^{3}}\right) \frac{W\left(\eta_{2}-b\right)}{\left(\eta_{1}-b\right)}
$$

where $\mathrm{W}(\mathrm{x})$ is the width of the Strait of Gibraltar at the surface in the different sections. If critical condition in CS is imposed $\left(G^{2}=1\right)$, then the expression (5) is readily obtained. Figure 3 shows the loci where the flow is supercritical (SPC, $G^{2}>1$ ) as a function of the different variables involved in 6 for
$Q_{1}=0.8 \mathrm{~Sv}, Q_{2}=0.8 \mathrm{~Sv}$ (that is, no barotropic flow) and $g^{\prime}=0.019 \mathrm{~ms}^{-2}$ (equivalent to a density difference of $2 \mathrm{~kg} \mathrm{~m}^{-3}$ ). When the interface lies inside the dark shaded area, the flow is SPC. Otherwise, it is subcritical (SBC, $\left.G^{2}<1\right)$. The minimum of the curve separating SPC and SBC regions is obtained in TN, as expected.

The extension of the shaded areas is sensitive to the parameter values. Obviously, the shaded area near the surface is achieved by means of the first term within brackets in 6 . It is sensitive to $Q_{1}$ in the sense that if it increases, the shaded area also does (the new boundary of the upper SPC region descends to the line labelled "a" in Fig. 3) but it is not sensitive to changes in $Q_{2}$. It is the shaded area near the sea bottom that is sensitive to $Q_{2}$ variations (line labelled "b" becomes the new boundary of the lower SPC region if $\left|Q_{2}\right|$ increases). Both areas are equally sensitive to changes of the density differences ( $\left.\mathrm{g}^{\prime}\right)$ : the less the density difference the greater the size of the shaded areas. For a value of the density difference low enough, both shaded areas will start merging in CS section, in which case the flow will be necessarily controlled at CS.

Curve " 1 " in Figure 3 is always inside the SBC region for the above mentioned values of the parameters, indicating that neither TN nor CS control sections exist. On the contrary, curve " 2 " gives critical flow at CS (the initial assumption in this work) and subcritical elsewhere eastward of this section. If $\mathrm{Q}_{1}$ is increased, the other parameters remaining unchanged, the upper SPC region extends downward and the situation "2" can become critical at TN. Curve " 3 " corresponds to a situation with the flow controlled at CS and at TN, and SPC flow east of TN. This is the typical picture of the maximal exchange.

\section{Physical processes forced by the boundary condition}

The boundary condition 5 is an essential condition to the model solutions. It determines the interface depth if the density contrast and the geometry of the Strait are given. From all solutions mathematically feasible in the case of triangular geometry, only two situations are physically meaningful. In terms of Figure 3, condition 5 implies that the interface must lie inside one of the two SPC regions at CS. One possibility is that the interface is inside the lower shaded area, in which case the model solution indicates that the interface depth throughout the 

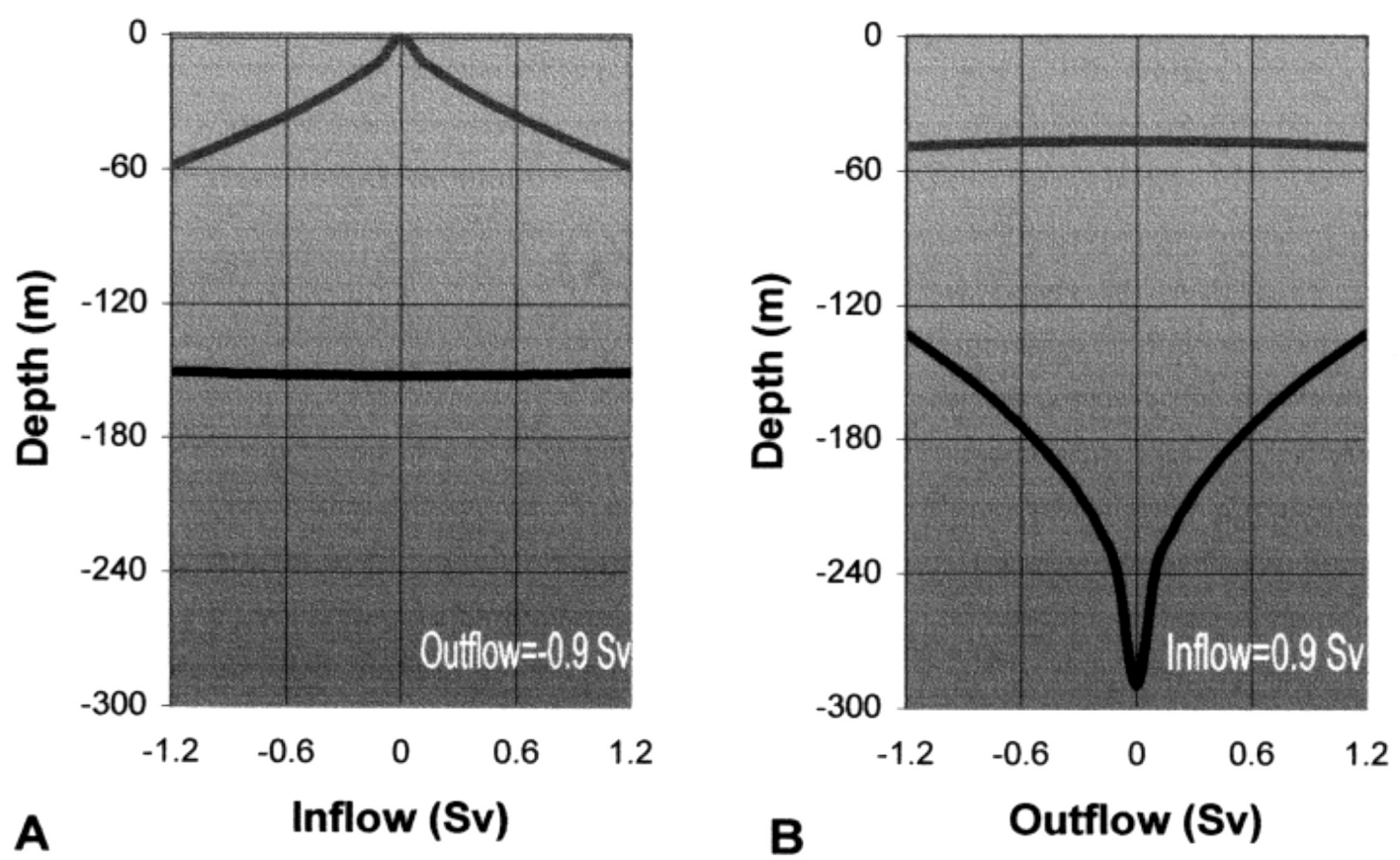

FIG. 4. - A) Depth of the interface at CS for the two physically meaningful solutions commented on the text as a function of $\mathrm{Q}_{1}$ and constant outflow. The upper curve represent the depth that the interface should have in the case that the critical condition were achieved through a fast-flowing (active) upper layer (and, therefore, a sluggish lower layer). The lower curve should be the depth of the interface in the case that the critical condition is achieved by means of a fast-flowing (active) lower layer (and a correspondingly sluggish upper layer). B) Depth of the interface as a function of $\mathrm{Q}_{2}$ and constant inflow. The caption of this panel follows that of panel A). In both cases, a triangular cross-section has been assumed. As argued in the text, the physically acceptable situation corresponds to the lower curves, that is, an active lower layer.

Strait depends almost exclusively on the values of the outflow $Q_{2}$ (see Fig. 4A). Only when the exchange is close to maximal, the depth of the interface is also sensitive to $Q_{1}$, particularly in the vicinity of TN. The second possibility would correspond to a fast flowing upper layer with a shallow interface (upper shaded area in Fig. 3, see also Fig. 4B). The topography of the Strait (with the narrowest section east of the sill section) along with the cascading of the Mediterranean outflow down to $1000 \mathrm{~m}$ depth into the Atlantic Ocean make us to reject the second possibility. Therefore, the interface depth along the Strait is mainly sensitive to the accepted value of $Q_{2}$.

The depth of the interface also depends on the density contrast $\Delta \rho$ and on the net barotropic flow $Q_{0}$. However, as far as the exchange is submaximal, the interface depends on $Q_{2}$ almost uniquely. Figure 5 (black lines) shows that if we solve the model for a given value of $Q_{1}$ and allow for variations of $\Delta \rho$ and $Q_{0}$, then the interface moves up as $\Delta \rho$ diminishes and it moves down as $Q_{0}$ increases. This is so because an increase of the net barotropic flow $Q_{0}$ keeping $Q_{1}$ constant is only possible if $\left|Q_{2}\right|$ diminishes. A reduction of the outflow is followed by a deepening of the interface at CS in order to keep the critical condition and, hence, by a generalised sinking of the interface all over the Strait. This close dependence on $Q_{2}$ is better illustrated in Figure 5 (grey lines), which is the counterpart of the black lines on the same figure with $Q_{2}$ playing the role of $Q_{1}$. If the outflow is constant, the interface remains almost motionless for a wide range of values of $Q_{0}$.

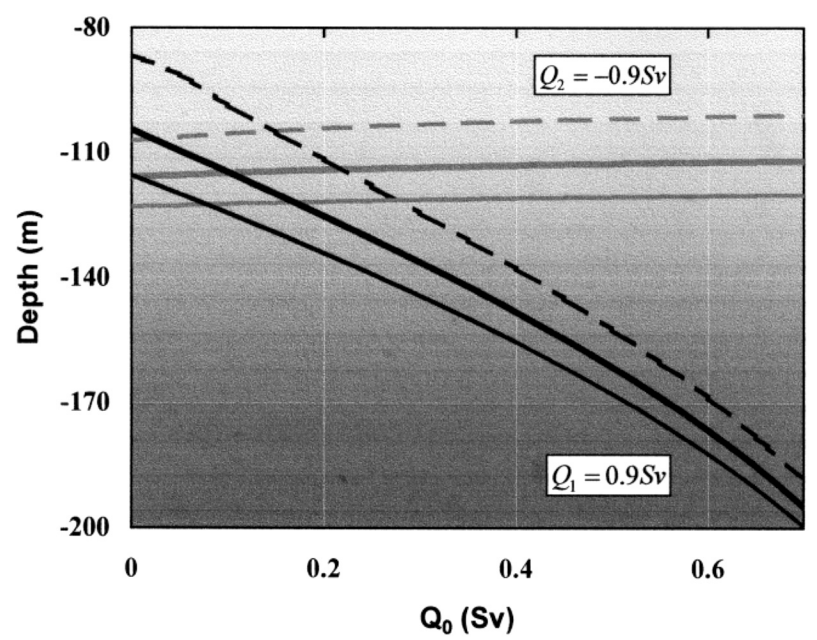

FIG. 5. - Interface depth at the eastern section of the Strait as a function of the net barotropic flow $Q_{0}$ and for different values of $\Delta \rho$ (or g') for two distinct situations. Black lines corresponds to a constant inflow of $0.9 \mathrm{~Sv}$ with the following style codes: thick line is for $\Delta \rho=2 \mathrm{~kg} \mathrm{~m}^{-3}$, thin line is for $\Delta \rho=2.5 \mathrm{~kg} \mathrm{~m}^{-3}$ and dashed line is for $\Delta \rho=1.5 \mathrm{~kg} \mathrm{~m}^{-3}$. Grey lines represent the situation of constant outflow of $-0.9 \mathrm{~Sv}$, with line style having the same meaning as in the previous case. 


\section{Barotropic fluctuations}

\section{General remarks}

Up to now, we have used prescribed values of flows in both layer and the density difference to determine the interface depth throughout the Strait. The net barotropic flow is obtained from the values of the exchanged flows. However, from an experimental point of view it is advisable to change the inputs of the model.

The net barotropic flow can be determined indirectly from the Mediterranean Sea response to external climatic forcing. Density difference fluctuations can be estimated independently because it is linked to the solar cycle. On the contrary, interface depth is difficult to determine from observations, because of the ambiguity of its definition (a two layer sea is a conceptual simplification of the reality). Direct surveys of this variable are scarce. Therefore, it seems adequate to prescribe the annual cycles of $Q_{0}$ and $\Delta \rho$ and then to analyse the behaviour of $\eta_{2}$. It is worthy to remark that the knowledge of $Q_{0}$ does not determine the size of the exchanged flows, which would remain unknown. To solve the problem, we need to prescribe another variable. Thus, we test a situation in which $\eta_{2}$ cycle is known and we observe the response of $Q_{1}$ and $Q_{2}$

\section{Experimental inputs}

The mass budget within the whole Mediterranean Sea would be written as:

$$
Q_{0}-(E-P)=d V / d t
$$

where V is the Mediterranean Sea volume. On average, this equation gives the well-known relationship $Q_{0}=(E-P)$ since $V$ does not change over long periods. However, on a seasonal scale, the former equation may have a RHS different from zero (see Ayoub et al., 1998), which indirectly imply a seasonal cycle for $\mathrm{Q}_{0}$ that can be obtained as $(E-P)+d V / d t$. Taking into account the steric effect, García Lafuente (2000) found a $\mathrm{Q}_{0}$ seasonal signal of 0.08 to $0.09 \mathrm{~Sv}$ of amplitude and around $220^{\circ}$ of phase (maximum value at year-day 224). These values are fully compatible with the observations of $Q_{0}$ reported in GLD01. Thus, we assume a time-dependent barotropic flow of the form

$$
Q_{0}=0.05+0.09 \cos (0.98 t-220)
$$

which has a mean value of $0.05 \mathrm{~Sv}$ (corresponding to a net evaporation of $0.6 \mathrm{~m} /$ year).

The difference of density $\Delta \rho=\rho_{2}-\rho_{1}$ have a clear annual signal induced by the seasonal cycle of solar heating. The lower layer density, $\rho_{2}$, remains rather constant since this water is not exposed to direct solar heating. Only the upper layer density changes appreciably, inducing the seasonal cycle of $g$. Historical records of temperature and salinity in the neighbourhood of the Strait (Gulf of Cádiz and Alborán Sea) collected during several decades indicate a seasonal cycle of $\Delta \rho$ given by (see Bormans et al., 1986):

$$
\Delta \rho=2.4+0.3 \cos (0.98 t-238)
$$

The signals 8 and 9 will be used as inputs for the model. It should be remarked that they are timedependent signals. However, as discussed earlier, the time scale of variability is so low that the stationary approach is justified and the time dependence of the solutions is achieved through the time dependence in Equations 8 and 9.

\section{Interface modelling}

The prescription of the barotropic cycle does not give information on whether it is a consequence of a variation of $Q_{1}$ or of $Q_{2}$, or of a combination of both. A simple answer is not possible because of the dependence of the interface depth solution on several parameters.

Let us consider a motionless interface throughout the year, that is, no seasonal signal in the interface depth. Then, the cross-areas do not change with time. This situation links barotropic fluctuations directly with the velocities of either layer, which shall be proportional to the density difference. In this case, the imposition of a net barotropic signal in the model leads to an enhanced response of $Q_{1}$ that accounts for most of the prescribed net barotropic flow. $Q_{2}$, which is also influenced by $\Delta \rho$, only smoothes the fluctuations of $Q_{1}$ to produce the barotropic signal (see Fig. 6C). Of course, the actual values of the flows depend on the constant depth assigned to the interface at, say, the eastern section. The shallower (deeper) the interface the greater (smaller) the exchanged flows.

To analyse the case in which the interface has a seasonal cycle, we must prescribe both the amplitude and the phase of the signal. Under submaximal exchange, the depth of the interface in the Mediter- 

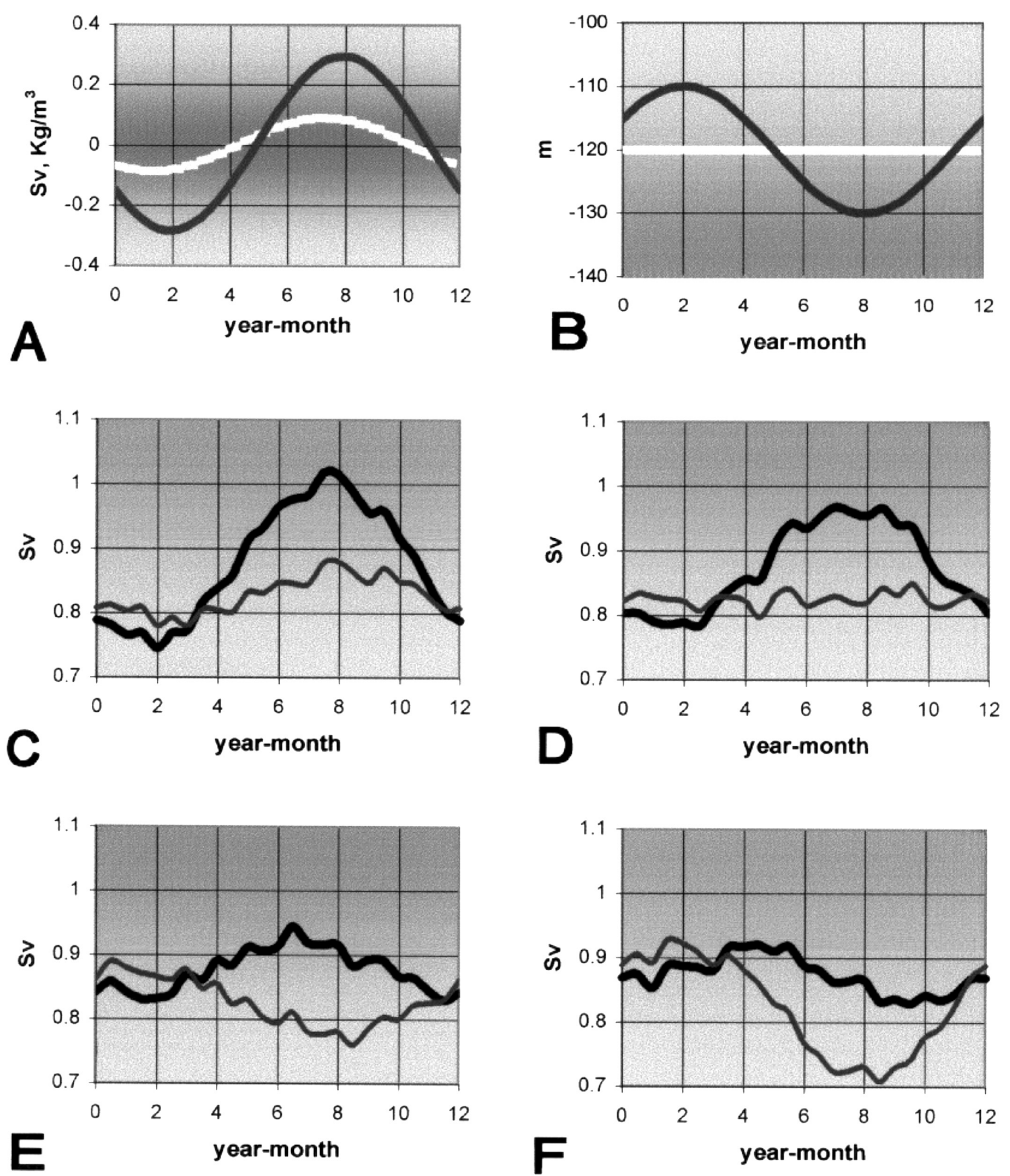

FIG. 6. - A) Accepted seasonal cycles for the net barotropic flow (white line) and for the density difference (grey line). B) Cycle of the interface depth at the eastern section of the Strait used to test the model. White line is for no cycle and grey line only indicate the phase of the cycle, since the amplitude changes from one test to the other. C) Inflow (black line) and modulus of the outflow (grey line) produced by the model when the amplitude of the interface oscillation is null (fixed interface). D), E) and F) Outputs of the model for interface oscillation amplitudes of $4 \mathrm{~m}$ (panel D), 10m (panel E) and $15 \mathrm{~m}$ (panel F). Always, black (grey) line represents inflow (outflow).

ranean side of the Strait has influence on the exchanged flows. GLD01 speculate with the fact that the increased internal radius of deformation during summer due to the density annual cycle produces larger and more stable anticyclonic gyre in the Western Alboran Sea, sinking the interface in this basin. If so, the interface in the Strait must be deeper in summer and shallower in winter, in agreement with the conceptual model of Bormans et al. (1986) in which the interface rises during winter due to the filling of the Mediterranean deep reservoir by the newly formed Mediterranean deep water. Observations taken in the eastern side of the Strait during CANIGO, reported in GLD01, give support to this temporal pattern. These authors report a seasonal cycle of the interface that peaks at $61^{\circ}$ (maximum 
interface height and minimum inflowing cross-area in year-day 62), which implies a maximum inflowing cross-area in summer $\left(241^{\circ}\right.$ of phase). We assume this value and analyse the sensitivity of the exchanged flows to interface amplitude variations at the eastern section of the Strait.

For moderate amplitudes (less or around $5 \mathrm{~m}$ ), the seasonal signals of the inflow $Q_{1}$ and the modulus of the outflow $\left|Q_{2}\right|$ decrease (their difference being $Q_{0}$ ) but still oscillate in phase (see Fig. 6D). As in the zero-amplitude situation, barotropic fluctuations are explained by the inflow oscillations. As we increase the amplitude of the interface oscillation, the outflow start oscillating in phase opposition with the inflow (which still oscillates in phase with $Q_{0}$ ) and the amplitude of the inflow oscillation diminishes (Fig. 6E). An increase of $Q 1$, and a diminution of $Q 2$ that are comparable in magnitude produce the barotropic signal. If we still increase the amplitude of the interface oscillation, then the barotropic fluctuation is achieved by a diminution of the outflow, the inflow remaining less sensitive to the seasonal signal (Fig. 6F).

Of all the situations illustrated in Figure 6, the case in panel D matches best the scarce observations of the interface oscillation taken at the eastern exit of the Strait (see GLD01). Thus, the seasonal cycle of the net barotropic flow would arise from an in phase increment of the inflow and a much less important fluctuation of the outflow. This mechanism is also put forward by GLD01 when interpreting the CANIGO data acquired in the eastern section of the Strait. In the model, this behaviour is a consequence of the presence of the critical section at CS, which imposes an additional restriction to the outflow variations. The inflow, however, can easily follow the fluctuations of the driving forces as far as it does not reach critical conditions in the second control section.

The ultimate reason of the interface oscillation at the eastern section remains unclear. Under submaximal exchange, it may be connected to the conditions in the Alboran Sea basin. Anyway, the linking of this oscillation with the size and permanence of the Western Alboran Gyre during summer needs further experimental confirmation.

\section{Other barotropic looks}

There are other issues that can be addressed by this simple model. One of them is to find which section of the Strait would be more sensitive to interface oscillation when a barotropic fluctuation is imposed. The frictionless model indicates that this section is situated $23,65 \mathrm{~km}$ eastward of CS, just in TN (minimum width section), as it can be expected from the Strait geometry. If friction is included, the model predicts an slight eastward displacement of this section (no more than $1 \mathrm{~km}$ for realistic values of the drag coefficients $C_{i}$ and $C_{b}$ in Equations 2 and 3 ) and its localisation is rather independent of the barotropic fluctuation imposed. For instance, with net barotropic fluctuations between -0.1 and $0.4 \mathrm{~Sv}$ and with a constant outflow of $-0.84 S v$ (that forces the inflow to change in order to explain the barotropic fluctuation), the greatest displacement of the section of maximum sensitivity is $0.88 \mathrm{~km}$ (24.53 km east of CS) for drag coefficients of $C_{i}=2$ $10^{-4}$ and $C_{b}=410^{-3}$. This result agrees with other previously obtained (Pratt, 1986; BG89).

Another interesting question is how the barotropic fluctuation is achieved if the exchange is maximal. The new constraint that arises (control section at $\mathrm{TN}$ ) reduces by one the number of independent variables and establishes upper limits for the exchanged flows. In this case, a reduction of the outflow (modulus) and a quite similar increase of the inflow achieve the positive barotropic fluctuation. In practice, inflow and outflow would obey the rules, $Q_{1}=\overline{Q_{1}}+Q_{0} / 2, Q_{2}=\overline{Q_{2}}+Q_{0} / 2$, (overbar denote mean values in absence of barotropic fluctuation). This is significantly different in the case of submaximal exchange (see Fig. 6) and indicates that under maximal exchange a barotropic fluctuation forces $Q_{1}$ and $Q_{2}$ signals to be coupled.

\section{DISCUSSION AND CONCLUSIONS}

Some results about the seasonal signal in the exchanged flows obtained from data collected during CANIGO and analysed in GLD01 indicate that maximal exchange is not compatible with the observations. GLD01 report a seasonal cycle of the inflow that is not coupled with a similar cycle in the outflow, neither with the interface oscillation. We have just seen that this behaviour cannot be explained in a maximal exchange (double hydraulic control) situation. Physically, if we assume $g^{\prime}$ constant, an increase of $Q_{1}$ must be followed by a deepening of the interface at TN in order to keep the critical condition there. The perturbation would freely progress westward sinking the interface at CS and reducing the outflow in order to maintain the critical condition at CS. Thus, inflow, outflow, and interface 
depth would have phase-locked cycles. If we allow for $g^{\prime}$ variations, the coupling is more evident: both flows are proportional to $g^{1 / 2}$. The absence of one control section allows the flows to oscillate independently without violation of the internal critical conditions. We have worked out the hypothesis that TN control does not exist and, therefore, the exchange is submaximal. Under this hypothesis, the conditions in the Alboran Sea effectively influence the exchange: a deeper interface in this basin will enhance the inflow and hamper the outflow (the opposite, if the interface rises).

Our numerical model explores this submaximal situation. Once the channel geometry and, eventually, the friction coefficients have been specified, the interface depth, $\Delta \rho, Q_{1}$, and $Q_{2}$ are not independent (since $Q_{0}=Q_{1}+Q_{2}$, we can use $Q_{0}$ and $Q_{1}$, (or $Q_{2}$ ) instead of $Q_{1}$ and $Q_{2}$ in the above set of variables). To run the model, we have to prescribe three of these variables. The net barotropic flow is one of them (Eq. $8)$. The density difference is the second prescribed variable (Eq. 9). We still need to prescribe a third variable (interface depth or one of the two flows) to solve the problem. At this point, we have run the model with different values of the interface depth at the eastern section to obtain the exchanged flows. The results, summarised in Figure 6, show that a time-independent interface implies a dominant role of $Q_{1}$ in order to explain the prescribed barotropic signal. As the amplitude of the seasonal signal of the interface increases, $Q_{2}$ becomes more important in the barotropic signal. For the observed amplitudes of around $5 \mathrm{~m}$ (see GLD01), the inflow fluctuations still dominate the barotropic signal. The model output agrees with the idea that the annual cycle in $Q_{0}$ is originated by a cycle in $Q_{1}$ driven by the solar cycle. $Q_{2}$ would remain less sensitive to this cycle because the seasonal signals of the interface oscillation and of $\Delta \rho$ act in opposite directions: in summer, when the interface is deeper (less outflow because of the hydraulic control at CS), $\Delta \rho$ is greater (more outflow because of an enhanced driving force).

The role of friction in the model has been also analysed. Using the quadratic laws of equations 2 and 3 with realistic numerical values of drag coefficients, friction slightly flattens the interface shape along the Strait in the case of submaximal exchange. Interfacial friction is noticeable near $\mathrm{TN}$ because of the sensitivity of the interface depth to changing conditions at this key section. Under maximal exchange, interfacial friction displaces the critical section of TN towards the east, a fact that is explained by the friction-induced sinking of the interface, which is now deeper than in the frictionless case at TN. Friction with walls is more pronounced at CS and deepens the interface throughout the Strait.

The model can be improved taking account of the water entrainment that the active layer produces over the more passive one. If we define inflow and outflow as the amount of water entering and exiting the Mediterranean Sea, this entrainment makes these flows to change along the Strait. Estimates of these variations can be made applying the mass and salt conservative budgets for the Mediterranean (the well-known Knudssen relationships). They combine to yield the equation

$$
Q_{2}=\frac{E \cdot S_{1}}{\Delta S}
$$

where $\Delta S$ is the salinity difference and $\mathrm{S}$, is the Atlantic water salinity. Since $\Delta S$ diminishes in the Strait due to water entrainment and mixing, the outflow (and, hence, the inflow) increases. Rough estimates point at a $10 \%$ increase on average. Therefore, the interface would descend about $6 \mathrm{~m}$ at the eastern section. This variable flow can be easily integrated in the model, but the modifications it induces are not of much concern for the results we present here.

To conclude, the model hints at $\mathrm{TN}$ section as a key site for monitoring the exchanges. This is true not only for maximal exchange but also for submaximal exchange and its low frequency variability. It is the most sensitive section for interface depth variations induced by a fluctuating forcing.

\section{ACKNOWLEDGEMENTS}

This work has been partially supported by the European Union Project Canary Islands Azores Gibraltar Observations (CANIGO), under contract MAS3 CT096 and by Spanish Comisión Interministerial de Ciencia y Tecnología (CICYT) project MAR96-1950-CO2-01. We are grateful to our CANIGO colleagues for much helpful discussion on the water exchange through the Strait.

\section{REFERENCES}

Armi, L. and D.M. Farmer. - 1988. The flow of Mediterranean water through the Strait of Gibraltar (see also Farmer D. M. and L. Armi. - 1988. The flow of Atlantic water through the Strait 
of Gibraltar), Progress in Oceanogr., 21: 1-105.

Ayoub, N., P.Y. Letraon and P. de Mey. - 1998. A description of the Mediterranean surface variable circulation from combined ERS-1 and TOPEX/POSEIDON altimetric data. J. Mar. Systems, 18: 3-40.

Baringer M. and J.F. Price. - 1997. Mixing and Spreading of the Mediterranean Outflow. J. Phys. Oceanogr., 27: 1654-1675.

Bormans, M., C. Garrett and K.R. Thompson. - 1986. Seasonal variability of the surface inflow through the Strait of Gibraltar. Oceanol. Acta., 9: 403-414.

Bormans, M. and C. Garrett. - 1989. The effects of rotation on the surface inflow through the Strait of Gibraltar. J. Phys. Oceanogr., 19: 1535-1542.

Bormans, M. and C. Garrett. - 1989. The effects of nonrectangular cross section, friction and barotropic fluctuations on the exchange through the Strait of Gibraltar, J. Phys. Oceanogr., 19: 1543-1557.

Bryden, H.L., J. Candela and T. H. Kinder. - 1994, Exchange through the Strait, of Gibraltar, Progress in Oceanogr., 33: 201-248.

Bryden, H.L. and T. Kinder. - 1991. Steady two-layer exchange through the Strait of Gibraltar. Deep-Sea Res., 38S: S445-S463.

Chapra and Canale. - 1988. Métodos numericos para ingenieros. Ed. McGraw-Hill. México.

Farmer, D.M. and L. Armi. - 1986. Maximal two-layer exchange over a sill and through the combination of a sill and contraction with barotropic flow. J. Fluid Mech., 164: 53-76.

Garcia Lafuente, J., J.M. Vargas, J. Candela, F. Plaza, B. Bascheck and T. Sarhan. -2000 . Tide at the eastern section of the Strait of Gibraltar. J. Geophys. Res., 105: 14197-14213.

Garcia Lafuente, J., J. Delgado, J.M. Vargas, M. Vargas, F. Plaza, T. Sarhan. - 2001. Low frequency variability of the exchanged flows through the Strait of Gibraltar during CANIGO. Deep Sea Res. (in press).

García Lafuente, J. - 2000. Consideraciones sobre los flujos intercambiados a través del Estrecho de Gibraltar. Variaciones estacionales. In: J.J. Alonso and F. Ortegon (eds.), Proceedings on the Second Workshop on exchanged flows through the Strait of Gibraltar, Cadiz (Spain).

Garrett, C. - 1996. The role of the Strait of Gibraltar in the evolution of Mediterranean water, properties and circulation. In Dynamics of the Mediterranean Straits and Channels F. Brian ed., CIESM Science Series No 2, 1-19.

Helfrich, K.R. - 1995. Time-dependent two-layer hydraulic exchange flows. J. Phys. Oceanogr., 25: 359-373.

Pratt, L.J. - 1986. Hydraulic control of sill flow with bottom friction. J. Phys. Oceanogr., 16, 1970-1980.

Watson, G. and I.S. Robinson. - 1990. A study of internal waves propagation in the Strait of Gibraltar using shore-based marine radar images. J. Phys. Oceanogr., 20: 374-395.

Scient. ed.: J. Font 